\title{
Mixed or immune complex cryoglobulinaemia and neuropathy
}

\author{
J. J. CREAM ${ }^{1}$, J. E. C. HERN ${ }^{2}$, R. A. C. HUGHES ${ }^{3}$, \\ AND I. C. K. MACKENZIE \\ From the Department of Dermatology, Middlesex Hospital, \\ and the Department of Neurology, Guy's Hospital, London
}

SUMMARY Three patients with peripheral neuropathy and mixed or immune complex cryoglobulinaemia are reported. The significance of mixed cryoglobulinaemia and the pathogenesis of the peripheral neuropathy are discussed.

Cryoglobulins are serum proteins that precipitate in the cold and redissolve on warming. They may be of two types: (1) single component, made up of a monoclonal immunoglobulin, or (2) mixed, in which at least two different immunoglobulins are present, one being an antibody against the other. Single component cryoglobulins occur in myeloma, Waldenström's macroglobulinaemia, and as a benign monoclonal gammopathy. Mixed cryoglobulins have been described in rheumatoid arthritis, systemic lupus erythematosus, lymphomas, and several infections (Cream, 1972a). Often no cause is established.

Peripheral neuropathies have been reported in some patients with cryoglobulinaemia (Garcin et al., 1957, 1960; Boudin et al., 1959; Charmot et al., 1959; Siguier et al., 1964; Dayan and Lewis, 1966; Meltzer et al., 1966; Logothetis et al., 1968). In their review of the literature, Logothetis et al. (1968) found reports of a neuropathy in nine out of 137 patients with cryoglobulinaemia, an incidence of $7 \%$. Of the 12 patients of Meltzer et al. (1966) with cryoglobulinaemia, only one had a neuropathy. The neurological findings have varied. In most, both motor and sensory fibres have been involved. Tendon reflexes in the legs may be absent (for example, Siguier et al., 1964) or normal (for

1 Present address: Mayday Hospital, Thornton Heath, Surrey CR4 7YE.

2 Present address: Aberdeen Royal Infirmary, Foresterhill, Aberdeen AB9 2ZB.

3 Present address: National Hospital for Nervous Diseases, Queen Square, London W.C.1. example, case 1 of Logothetis et al., 1968). Cranial nerves may be involved (Logothetis et al., case 2) and the plantar responses extenso $\bar{B}$ (Logothetis et al., case 2). The protein of the

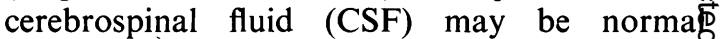
(Dayan and Lewis, 1966) or raised (Siguier et al 1964).

Most of these cases were reported before techniques were available for characterizing $\overrightarrow{0}$ cryoglobulins, and it is likely that they are $\vec{a}+\vec{f}$ heterogeneous group, which might explain the varying neurological picture. We report on three patients with neuropathies and cryoglobulinaemia in which the cryoglobulin has been identified and was of the mixed type. Two have not been described before. The third was described by Dayan and Lewis (1966) but at that time the cryoglobulin had not been typed.

\section{CASE 1}

This 67 year old housewife had had recurrent haemoptyses for about 15 years. Investigation at various chest clinics had not revealed a cause. Over the same period she had had Raynaud's phenomenon which, by 1964 , necessitated bilateral cervical sympathectomy - with only transient improvement. In 1967 purpura-mainly over the lower limbsfirst appeared. A diagnosis of allergic vasculitis was made and she was started on prednisone $15 \mathrm{mg} / \mathrm{day}$. This did not influence her purpura, which has persisted. It is usually worse in the summer. In 1969 she noticed numbness of the legs up to the knees and numbness of the fingers of the left hand. In 1970 she 
had the first of several attacks of loss of consciousness, preceded by a feeling of strangeness lasting from a few seconds to two minutes.

She was admitted to hospital in October 1970. On examination she had purpura over the legs and arms with ankle oedema. Neurological examination of the arms was normal. Hip flexion, extension of the big toes, and flexion of the toes were slightly weak. The ankle reflexes were absent. All the other tendon reflexes were present and the plantar responses were flexor. There was dense impairment to all modalities of cutaneous sensation up to the mid-calf, and some impairment to below the knee in a stocking distribution. Joint position and vibration sensation were normal.

Investigations included: erythrocyte sedimentation rate $20 \mathrm{~mm}$ in first hour (Westergren), haemoglobin $12.0 \mathrm{~g} / 100 \mathrm{ml}$., blood leucocytes $5,000 /$ $\mathrm{cu}$. $\mathrm{mm}$ and platelets $110,000 / \mathrm{cu}$. mm, sheep cell agglutination titre $1 / 128$, antinuclear factor (ANF) 1/160 (membranous), serum protein electrophoresis - low gamma globulin, serum IgG $760 \mathrm{mg} / 100 \mathrm{ml}$. (normal $950-1,500$ ), serum IgA $330 \mathrm{mg} / 100 \mathrm{ml}$. (normal 165-410). Monomeric 7S IgM was present in the serum, so that the amount of IgM could not be measured accurately. Serum cryoglobulin was $305 \mathrm{~g} / \mathrm{ml}$. (normal <80, Cream, 1972b) with IgG and IgM components. The following estimations gave normal or negative results: LE cells, skeletal survey, serum $\beta_{1} \mathrm{C} / \beta_{1} \mathrm{~A}$ globulin, WR, Australia antigen, serum $B_{12}$, and folate.

The bone marrow showed a slight increase in plasma cells with occasional atypical forms. A skin biopsy showed an acute allergic vasculitis.

Electrophysiological studies were performed by Dr. P. K. Thomas. Sampling of the left extensor digitorum brevis with a concentric needle electrode revealed a grossly reduced motor unit recruitment

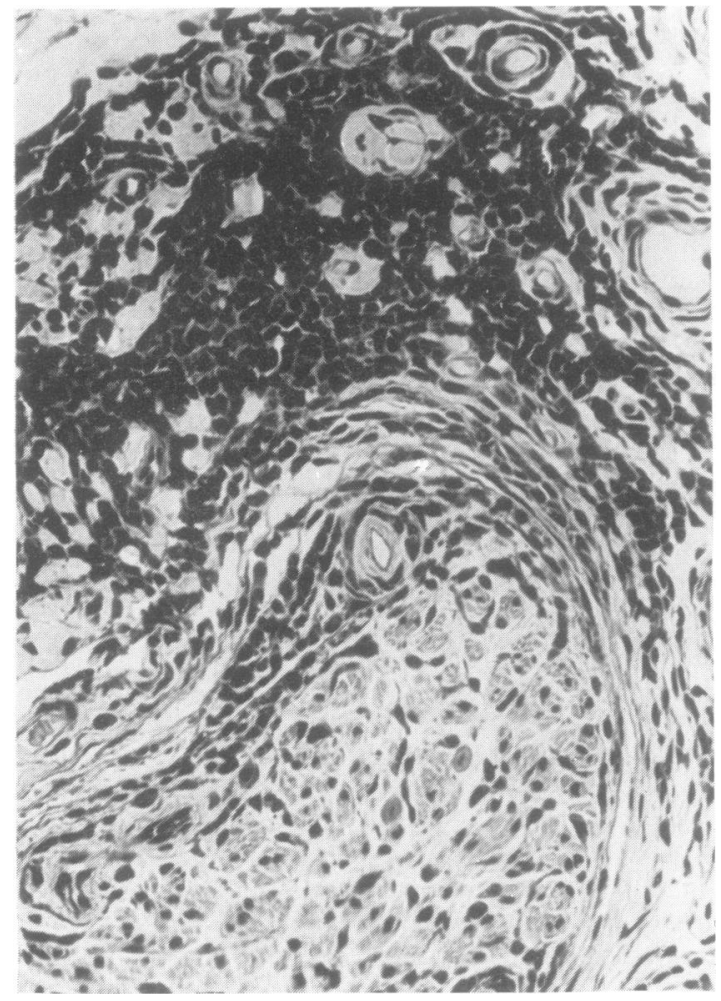

FIG. 1. Sural nerve biopsy (case 1) showing perivascular infiltration by lymphocytes and plasma cells; the cells are extending into the nerve bundle. $H$ and $E$, $\times 260$.

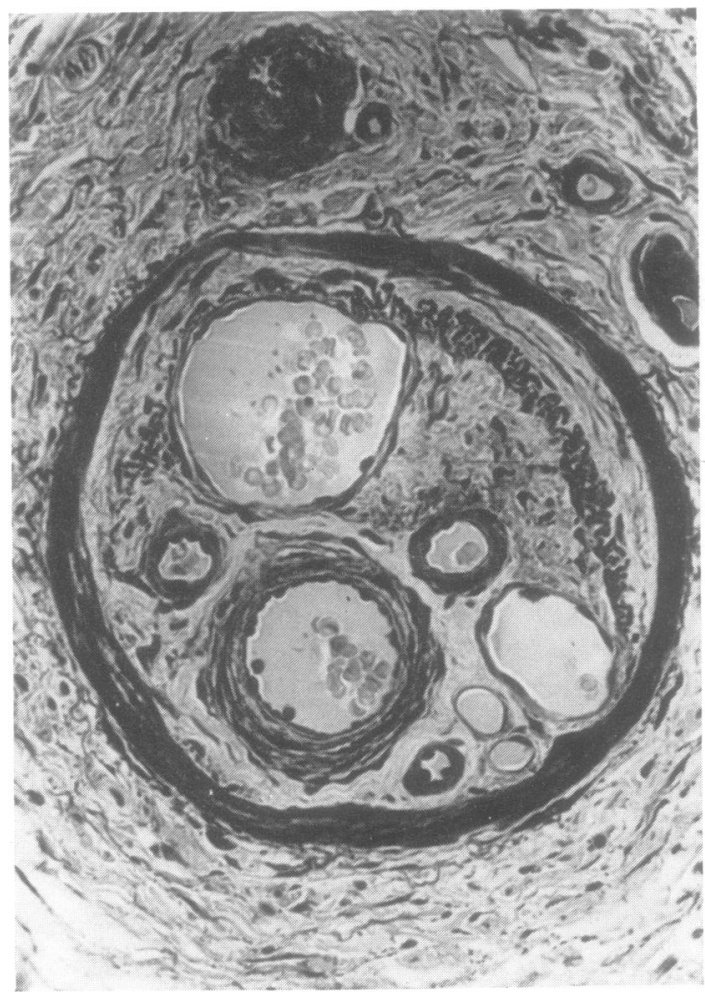

FIG. 2. Sural nerve biopsy (case 1) showing revascularization of a small artery with partial destruction of the internal elastic lamina. Verhoeff's stain for elastic fibres, $\times 500$. 
pattern. Surviving motor unit potentials were normal and fired at high rates on maximum voluntary effort. Motor nerve conduction velocity in the left lateral popliteal nerve was slightly reduced $(36 \mathrm{~m} /$ sec). No sensory nerve action potentials could be detected in the left median or ulnar nerves at the wrist.

A sural nerve biopsy (R. O. Weller) showed evidence of vasculitis with perivascular lymphocytes and plasma cells extending through the perineurium into the nerve fasciculi (Fig. 1). The internal elastic lamina of an epineurial artery had been partly destroyed and the occluded lumen revascularized (Fig. 2); this suggests healed vasculitis. There was extensive loss of myelinated nerve fibres due to axonal degeneration (Fig. 3). No evidence of amyloid deposition was found.

In April 1971 she was readmitted. For the previous month she had had considerable purpura and occasional attacks of colicky central abdominal pain

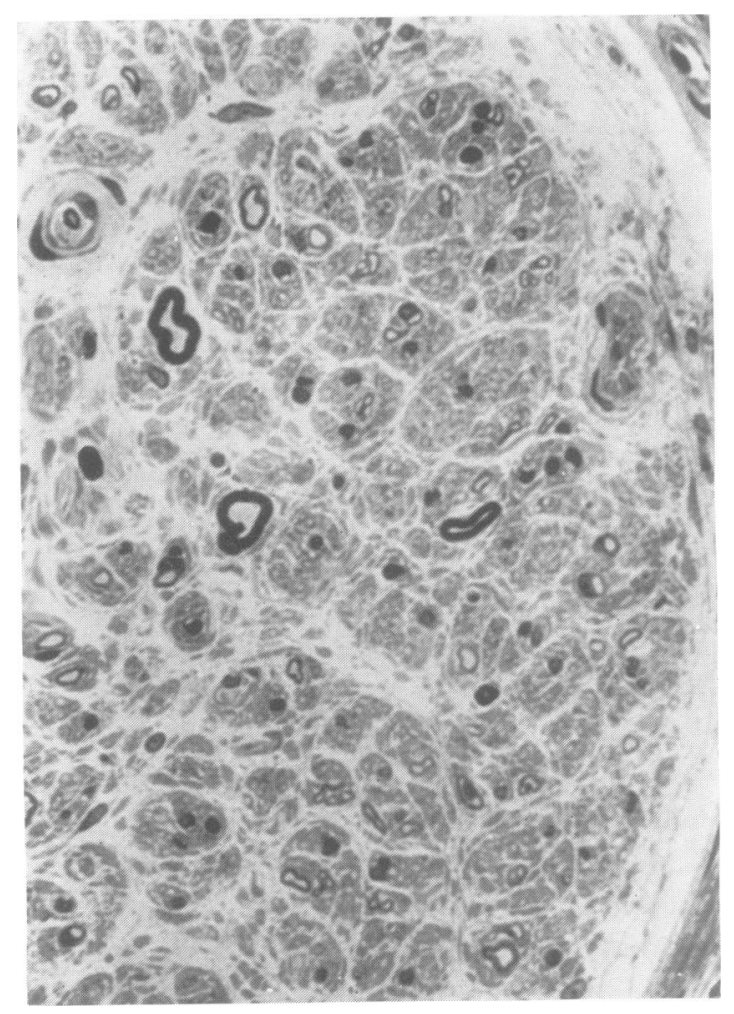

FIG. 3. Sural nerve biopsy (case 1) showing severe loss of large and small myelinated fibres. $1 \mu \mathrm{m}$ Araldite section stained with toluidine blue, $\times 560$. with diarrhoea. These attacks continued over the next few weeks, sometimes being associated with haematemesis and passage of blood per rectum. Barium meal and enema and sigmoidoscopy revealed no abnormalities. She was started on penicillamine capsules, initially $300 \mathrm{mg}$, increasing to $600 \mathrm{mg} /$ day. A month later, however, although her abdominal attacks had ceased, she had deteriorated in that her purpura was more extensive and she developed heart failure, proteinuria, and uraemia. Dexamethasone, digoxin, and diuretics were substituted for the penicillamine.

Examination in July 1971 showed severe weakness of both masseter muscles and of all muscle groups of both arms and legs. The ankle reflexes were absent, as before, but the plantar responses were now unobtainable. There was impairment of light touch sensation over all the digits of the left hand, and impairment of all modalities of cutaneous sensation over both legs up to the mid-thigh on the right side and the tibial tuberosity on the left side. Joint position sense was impaired at the distal interphalangeal joints of all the fingers of the left hand and at the metatarsophalangeal joints of both great toes.

In December 1971 her weakness had decreased but she was occasionally confused.

\section{CASE 2}

This 65 year old housewife presented in 1967 with one year history of purpura over the legs. A skin biopsy showed a leukocytoclastic vasculitis. In 1969 she had several haemoptyses, each coinciding with a fresh crop of purpura. Laryngoscopy revealed haemorrhagic crusts in the larynx. Her purpura ceased in 1969 but since 1968 she had noticed numbness of both feet as if she was walking 'on blocks', with tingling in the distal third of the feet.

Examination in 1971 showed weakness of eversion and dorsiflexion of the left ankle and of extension of the left great toe. The ankle reflexes were absent but the other reflexes were normal. There was no demonstrable sensory loss. Investigations in 1970 revealed a mixed IgG-IgM cryoglobulin in the serum $(160 \mu \mathrm{g} / \mathrm{ml}$.). Normal investigations included: sheep cell agglutination, LE cells, ANF, serum immunoglobulins, serum protein electrophoresis, and serum $B_{12}$ and folate.

Electromyography supported the clinical diagnosis of a peripheral neuropathy. The right extensor digitorum brevis muscle showed evidence of denervation on sampling with a concentric needle electrode. Positive sharp waves were present at rest and on maximum voluntary effort a few motor units fired at high rates in relative isolation. The right tibialis anterior muscle showed no abnormality. Maximum 
motor conduction velocity in the right common peroneal nerve was $51.8 \mathrm{~m} / \mathrm{sec}$ and the distal latency was $4.1 \mathrm{~m} / \mathrm{sec}$. No ascending nerve action potential could be recorded in the common peroneal nerve but the left ulnar and median sensory nerve action potentials were normal.

\section{CASE 3}

A report of this case including histological and electrophysiological studies has already been published (Dayan and Lewis, 1966) but, since the cryoglobulin has now been identified as a mixed cryoglobulin and the patient has now died, a further report seems desirable.

This woman presented in 1960 at the age of 53 years with Raynaud's phenomenon, tiredness, leg ulcers, and recurrent purpura over the legs, trunk, and arms. In 1963 a cryoglobulin was detected in the serum. A bone marrow biopsy was normal but when repeated one year later showed a slight lymphocytosis. An M-component in the $\gamma$-globulin region was noted on serum protein electrophoresis. Biopsy of a purpuric lesion revealed haemorrhage and necrosis around capillaries with dense infiltration by polymorphonuclear leukocytes. No cryoglobulin was seen in the vessels. In September 1963, she was started on prednisone $20 \mathrm{mg} /$ day. Three months later she was readmitted with a chest infection and slowly developed weakness of the left leg and a left foot drop. By April 1965 there was weakness of both legs especially the left, with impairment of all sensory modalities. The ankle jerks were absent and the knee jerks reduced. The arms were normal apart from impaired tendon reflexes. Electromyography confirmed the peripheral nerve damage and sural nerve biopsy showed severe demyelination with relative preservation of axons (Dayan and Lewis 1966). The CSF was normal. Prednisone was discontinued in 1966. In 1967 she was still troubled by recurrent purpura and leg ulcers but in addition had developed malignant hypertension which was subsequently controlled by chlorothiazide and methyldopa. Another marrow biopsy showed an increase in lymphocytes and plasma cells. It was noted at this time that, although the sheep cell agglutination test was negative, the latex test was strongly positive $(1 / 2,500)$ and analysis of the cryoglobulin showed that it contained IgM, IgG, and a small amount of IgA. In 1968 she ceased to have purpura but her leg ulcers never healed. After stopping her hypotensive therapy she developed a right-sided hemiplegia from which she recovered as her hypertension responded to treatment. In 1970 she developed gangrene of the right foot. Arteriography showed extensive arterio- sclerotic changes in the limb and an above knee amputation was performed.

At her final admission in June 1971, she complained of loss of appetite, diarrhoea, and right hypochondrial pain. Examination revealed generalized weakness, absent knee and ankle jerks, diminished tendon reflexes in the arms but no sensory impairment. She was hypertensive (blood pressure $200 / 145 \mathrm{mmHg}$ ) and had ulcers at the ankle. There was no purpura, lymphadenopathy, or hepatosplenomegaly. Investigation revealed no cause for her symptoms. Shortly after admission she became confused, febrile, and eventually comatose and died. Permission for a postmortem examination was refused.

\section{DISCUSSION}

A cryoglobulin may consist of a single component monoclonal immunoglobulin or it may be 'mixed' in that it contains two different immunoglobulins-one of which is an antibody against the other. The antibody component of a mixed cryoglobulin is a 'cold reactive' antibody which acts better in the cold than in the warm and so large precipitable antigen-antibody aggregates form on cooling and dissociate in the warm. The detailed physicochemical processes responsible for cryoprecipitation of single component cryoglobulins are obscure but must again involve the formation of large precipitable aggregates.

Single component cryoglobulins are produced by a clone of cells whose malignant nature is usually obvious as in multiple myeloma or Waldenström's macroglobulinaemia. Occasionally, the clone of cells is apparently benign, as indicated by the fact that there is no relentless increase in the serum level of the abnormal immunoglobulin. Mixed cryoglobulins occur in autoimmune disease and infections. They also occur in lymphomas where the tumour produces an immunoglobulin that happens to be a coldreactive antiglobulin antibody. Thus, both single and mixed cryoglobulinaemia may be associated with malignancy so that the pathogenesis of a neuropathy in a patient with cryoglobulinaemia could be secondary to the malignancy and unrelated to the presence of the cryoglobulin.

However, cryoglobulins may give rise to vessel damage and, indeed, all three of our patients had cutaneous vasculitis. In one patient 
there was histological evidence of vasa nervorum vasculitis. Whether vasculitis develops in cryoglobulinaemia depends on several factors. One effect of intravascular precipitation or gelling of a cryoglobulin is an increase in blood viscosity but hyperviscosity is probably relatively unimportant, since vasculitis is not a feature of the hyperviscosity syndrome. Much more important is the ability of certain aggregated immunoglobulins and immune complexes to activate the complement, kinin and clotting systems (Costanzi et al., 1969; Becker, 1971). Activation of the complement system, for example, leads to the production of a factor chemotactic for polymorphs and release of their proteolytic enzymes. If the aggregated immunoglobulins or immune complexes were coated on to or lodged in a vessel wall acute vasculitis would result.

The pathological properties of mixed cryoglobulins have been demonstrated convincingly. Whitsed and Penny (1971) noted haemorrhagic lesions after intradermal injection of a cryoglobulin in man, while McIntosh et al. (1971) observed glomerulitis after intravenous infusion of cryoglobulins in experimental animals.

The temperature at which cryoprecipitation occurs in vitro varies from one cryoglobulin to another. Rarely, a cryoglobulin may precipitate at $37^{\circ} \mathrm{C}$ (Sargent et al., 1970). Most cryoglobulins precipitate only at temperatures well below $37^{\circ} \mathrm{C}$, although cryoprecipitation can occur at room temperature and at temperatures found in the superficial skin vessels. At the higher temperatures encountered in deeper vessels, including the vasa nervorum, it is less likely that large cryoglobulin aggregates will be present. Smaller soluble aggregates-still possessing pathogenic properties-would, however, be present in these vessels if the antiglobulin antibody shows some activity at the higher temperature, or if immune aggregates have formed in the cooler skin vessels and have not completely dissociated by the time they reach the deeper ones.

Single component cryoglobulins are also capable of activating the complement system (Costanzi et al., 1969) which may account for the urticaria and purpura seen in some of the patients. With this type of cryoglobulinaemia vasculitis is usually confined to the skin.
Histological studies have been too few to justify any general conclusion about the changes occurring in damaged nerve fibres. In the first of the present cases, the sural nerve showed evidence of axonal degeneration but in their case 3 Dayan and Lewis (1966) reported 'demyelination' with axonal sparing. Where nerve conduction studies have been done (Dayan and Lewis, 1966; Logothetis et al., 1968; our cases 1 and 2), maximum motor conduction velocity has been normal or shown a slight fall which would be compatible with a loss of fast conducting fibres. They have not shown the gross slowing associated with severe widespread demyelination (Gilliatt, 1966).

It was pointed out above that peripheral nerve damage occurs in only a small proportion of patients with cryoglobulinaemia. The present three cases are from a series of 16 patients with mixed cryoglobulinaemia, a relatively high incidence of $19 \%$. The association may occu요 more frequently than has been recognized, for ir patients with cryoglobulinaemia the neuropathy $\vec{c}$ might be slight enough to be missed unless specifically sought. Conversely, the presence of cryoglobulins in the serum of patients witle neuropathy could readily be overlooked (Cream $\overrightarrow{0} \overrightarrow{0}$ $1972 \mathrm{~b})$. The possibility of mixed cryoglobul: aemia should therefore be considered when searching for the cause of an undiagnosed neuropathy.

Treatment of cryoglobulinaemia depends on the type of cryoglobulin and the cause. In mixed cryoglobulinaemia, infections and autoimmune disease need to be excluded before antimetabolic drugs are given in an attempt to suppress production of the 'cold reactive' antiglobulin antibody. When a lymphoma is secreting a coldreactive antiglobulin antibody, or a myeloma is producing a single component cryoglobulin, the symptoms may justify the use of antimetabolic drugs and even plasmapheresis.

We thank Dr. R. J. Cairns, Dr. R. H. Meara, and Dr. H. J. Wallace for permission to report on their patients, Dr. P. K. Thomas for permission to publish his electromyographic results, and Dr. R. O. Weller for his histological report. Part of this study was supported by funds granted by the Clinical Research Committee, Middlesex Hospital. 


\section{REFERENCES}

Becker, E. L. (1971). Nature and classification of immediatetype allergic reactions. Advances in Immunology, 13, 267313.

Boudin, G., Barbizet, J., and Dalloz, J.-C. (1959). Cryoglobulinémie et lympho-réticulo-sarcome. Observation d'un cas avec purpura nécrotique, syndrome de Raynaud, parotidite et névrite multiple. Presse Médicale, 67, 594-597.

Charmot, G., Laplane, G., André, L.-J., and Ferrand, J. (1959). Cryoglobulinémie essentielle avec érythème polymorphe et acroparesthésies. Presse Médicale, 67, 1939 1940.

Costanzi, J. J., Coltman, C. A., Jr., and Donaldson, V. H. (1969). Activation of complement by a monoclonal cryoglobulin associated with cold urticaria. Journal of Laboratory and Clinical Medicine, 74, 902-910.

Cream, J. J. (1972a). Immune complex disease. Cryoglobulins. Proceedings of the Royal Society of Medicine, 65, 274-275.

Cream, J. J. (1972b). Cryoglobulins in vasculitis. Clinical and Experimental Immunology, 10, 117-126.

Dayan, A. D., and Lewis, P. D. (1966). Demyelinating neuropathy in macrocryoglobulinemia. Neurology (Minneap.), 16, 1141-1144.

Garcin, R., Mallarmé, J., and Rondot, P. (1957). Cryoglobulinémie et névrite multiple des membres inférieurs. Revue Neurologique, 97, 142-146.

Garcin, R., Rondot, P., and Grupper, C. (1960). Cryo- globulinémie et périartérite noueuse à manifestations multinévritiques. Revue Neurologique, 103, 589-592.

Gilliatt, R. W. (1966). Applied electrophysiology in nerve and muscle disease. Proceedings of the Royal Society of Medicine, 59, 989-993.

Logothetis, J., Kennedy, W. R., Ellington, A., and Williams, R. C. (1968). Cryoglobulinemic neuropathy. Archives of Neurology (Chicago), 19, 389-397.

Mclntosh, R. M., Kulvinskas, C., and Kaufman, D. B. (1971). Cryoglobulins. 2. The biological and chemical properties of cryoproteins in acute post-streptococcal glomerulonephritis. International Archives of Allergy and Applied Immunology, 41, 700-715.

Meltzer, M., Franklin, E. C., Elias, K., McCluskey, R. T., and Cooper, N. (1966). Cryoglobulinemia-A clinical and laboratory study. 2. Cryoglobulins with rheumatoid factor activity. American Journal of Medicine, 40, 837-856.

Sargent, A. U., Saha, A., Klassen, G. K., and Rose, B. (1970). Studies of cryoprecipitation. 3. Hemodynamic and metabolic adaptation to a circulating single component cryoglobulin. American Journal of Medicine, 48, 54-62.

Siguier, F., Godeau, P., Lévy, R., Binet, J. L., and Hamida, B. (1964). A propos d'un cas de neuropathie cryoglobulinémique (étude clinique et biologique). Semaine des Hôpitaux de Paris, 40, 1928-1934.

Whitsed, H. M., and Penny, R. (1971). IgA-IgG cryoglobulinaemia with vasculitis. Clinical and Experimental Immunology, 9, 183-191. 